\title{
Das Arbeitsvertragsrecht in der Coronakrise
}

\author{
Stephan Klawitter
}

\section{Inhaltsübersicht}

A. Arbeitsvertragliche Leistungsrisiken in der Corona-Pandemie

B. Pandemiebedingte Arbeitsunfähigkeit/-unwilligkeit des Arbeitnehmers

I. Wegfall oder Beschränkung der Leistungsfähigkeit

II. Generelle Arbeitsunwilligkeit des Arbeitnehmers

III. Weigerung zur Durchführung einzelner Weisungen

C. Pandemiebedingte Schwankungen des Leistungsinteresses des Arbeitgebers

I. Ausfall oder Beschränkung des Leistungsinteresses

1. Die arbeitsvertragliche Betriebsrisikolehre

2. Reaktionsmöglichkeiten des Arbeitgebers

II. Steigerung des Leistungsinteresses

D. Besondere Vertragsdurchführungsrisiken $\quad 236$

I. Schutzpflichten des Arbeitgebers 236

$\begin{array}{ll}\text { II. Aufklärungspflichten } & 237\end{array}$

1. Aufklärungspflichten des Arbeitnehmers 238

2. Aufklärungspflichten des Arbeitgebers 239

E. Die Krisensicherheit des Arbeitsvertragsrechts in der Pandemie 239

I. Das Arbeitsvertragsrecht als Korridor interessengerechter Krisenbewältigung

II. Die arbeitnehmerähnlichen Selbständigen als Sorgenkinder der Pandemie

III. Die Pandemie als (verpasste) Chance für den Beschäftigungsmarkt

Die Coronakrise hat den deutschen Beschäftigungsmarkt fest im Griff. Praktisch jeder Arbeitgeber sieht sich in den vergangenen Wochen mit der Herausforderung konfrontiert, die innerbetrieblichen Abläufe gleichzeitig an die pandemiebedingten Vorsorgebedürfnisse einerseits und die verzerrten Absatzmarktverhältnisse andererseits anzupassen, ohne hierbei in Hysterie zu verfallen. Besonders für Arbeitnehmer sind diese Adaptionsmaßnahmen vielfach mit Unsicherheiten verbunden, da sie zahlreiche, bisher kaum erörterte Einzelrechtsfragen aufwerfen und das Vertrauensverhältnis zwischen der Belegschaft und dem Arbeitgeber als Krisenmanager auf eine Belastungsprobe stellen. Dies gilt umso mehr, da Arbeitnehmer auch jen-

* Der Autor ist Rechtsreferendar am Kammergericht Berlin und Lehrbeauftragter der Humboldt-Universität zu Berlin für Arbeitsrecht und Rechtsdidaktik. 
seits ihres Arbeitsverhältnisses auf krisenbedingte Veränderungen öffentlicher Infrastrukturen reagieren müssen und dabei nicht selten in Zielkonflikte mit den Interessen ihres Arbeitgebers geraten. Dabei bilden die Arbeitsvertragsparteien in der Pandemie gewissermaßen eine wirtschaftliche Schicksalsgemeinschaft, verfolgen sie doch zum Zwecke ihrer Existenzsicherung das gemeinsame Ziel, den Betrieb sicher durch die Krise zu navigieren.

Der nachfolgende Beitrag soll sich mit der Frage auseinandersetzen, wie es um die Krisensicherheit des Arbeitsvertragsrechts im Pandemiefall bestellt ist. Es soll beleuchtet werden, welche arbeitsvertraglichen Risiken durch den Ausbruch des Coronavirus ausgelöst oder vergrößert werden und welche Regelungen das Arbeitsrecht für eine interessengerechte Behandlung dieser Risiken bereithält. Dabei wird festzustellen sein, dass sowohl das Allgemeine Schuldrecht als auch das besondere Arbeitsvertragsrecht an zahlreichen Stellen abstrakte Wertungsentscheidungen treffen, die sich in der gegenwärtigen Krise trotz ihrer Neuartigkeit nutzbar machen lassen. Flankiert wird das Vertragsrecht dabei von öffentlich-rechtlichen Unterstützungsmaßnahmen für den Arbeitsmarkt, wobei stellvertretend das Kurzarbeitergeld zu nennen ist. Diese Maßnahmen sollen in Anbetracht des vertragsrechtlichen Charakters dieser Tagung hier nur insoweit Erwähnung finden, wie sie Auswirkungen auf das Rechte- und Pflichtenprogramm der Arbeitsvertragsparteien haben. ${ }^{1}$ Aus demselben Grund sollen auch kollektivrechtliche Fragestellungen, insbesondere solche über die Befugnisse des Betriebsrates in Pandemiezeiten, in dieser Untersuchung nur von untergeordneter Rolle sein. ${ }^{2}$

\section{A. Arbeitsvertragliche Leistungsrisiken in der Corona-Pandemie}

Will man sich einen Überblick über die pandemiebedingten Besonderheiten des Arbeitsvertragsrechts verschaffen, so blickt man zunächst auf ein hochdiverses Feld verschiedenartiger Fragestellungen in der gesamten Breite des individualarbeitsrechtlichen Problemspektrums. Die Coronakrise wirkt sich dabei nicht bloß auf Fragen der Entgeltfortzahlung und des Arbeitsschutzes aus, sondern strahlt auf zahlreiche arbeitsrechtliche Teildisziplinen wie das Kündigungsrecht, das Urlaubsrecht und sogar das Befristungsrecht aus. Hinzu kommt, dass sich die entstehenden Rechtsfragen in

1 Hierzu ausführlich C. Zieglmeier, DStR 2020, 729.

2 Zur Rolle des Betriebsrates in Pandemiefällen M. Lützeler, ARP 2020, 117. 
den seltensten Fällen abstrakt und losgelöst vom konkreten Inhalt des Arbeitsverhältnisses, von der konkreten Person des Arbeitnehmers sowie vom Geschäftsgegenstand des beschäftigenden Arbeitgebers beantworten lassen. „Es kommt darauf an“, droht mehr noch als sonst zu einer Standardantwort zu verkommen, die das Bedürfnis des Rechtssuchenden nach Sicherheit eher verstärkt denn befriedigt.

Umso wichtiger ist es, in einem ersten Schritt die wesentlichen vertraglichen Risiken, welche durch den Pandemiefall ausgelöst oder vergrößert werden, zu identifizieren und entlang dieser die rechtlichen Modifikationen des arbeitsvertraglichen Rechte- und Pflichtenprogramms zu systematisieren. Dabei lassen sich im Wesentlichen drei Leistungsrisikofaktoren des arbeitsrechtlichen Synallagmas erkennen, welche durch die Coronapandemie beeinflusst werden:

Erstens kann der Pandemiefall die Leistungsfähigkeit und -willigkeit des Arbeitnehmers vor besondere Probleme stellen. Das offensichtlichste Beispiel hierfür ist das durch die Infektionsgefahr gesteigerte Krankheitsrisiko des Arbeitnehmers, der im Fall einer Erkrankung als Arbeitskraft auszufallen droht. Die Pandemie vergrößert allerdings nicht nur bestehende und allgemein hinzunehmende Leistungsausfallrisiken, sondern schafft auch neue Gefahren für eine ordnungsgemäße Vertragsdurchführung: So müssen Arbeitnehmer, die Eltern erziehungsbedürttiger Kinder sind, aufgrund der Kita- und Schulschließungen die mit ihrer Arbeitspflicht kollidierenden Betreuungspflichten erfüllen. ${ }^{3}$ Besteht der Verdacht einer Infektion, können Behörden gem. $\$ 30$ IfSG den Arbeitnehmer in häusliche Zwangsquarantäne versetzen, was ihm ein Erscheinen im Betrieb unmöglich macht. Und nicht zuletzt können Arbeitnehmer - besonders solche, die als Risikokandidaten einer Infektion gelten - aufgrund der gegenwärtigen Gefahrenlage das Risiko scheuen, den Arbeitsplatz zu betreten, um sich nicht der Ansteckungsgefahr durch die Kolleginnen und Kollegen auszusetzen.

Zweitens kann das Interesse des Arbeitgebers am Erhalt der Leistung während der Pandemie und auch in der Folgezeit modifiziert sein. Verhängen die Ordnungsbehörden eine Schließungsverfügung für das Ladengeschäft des Arbeitgebers, wird er für die Dauer der Schließung auch dann kein Interesse an der Leistung seiner Verkaufsangestellten haben, wenn diese gesund und arbeitswillig sind. Das Leistungsinteresse kann aber auch

3 Nach Angaben des Statistischen Bundesamtes sind auf Grundlage der Zahlen von 2018 durch die Coronakrise 692.000 Alleinerziehende und 3,5 Millionen Paarfamilien mit Kindern unter 13 Jahren von Betreuungsengpässen bedroht, Pressemitteilung Nr. N 012 vom 17. März 2020, https://www.destatis.de/DE/Presse/Pressemitte ilungen/2020/03/PD20_N012_122.html (zuletzt abgerufen am 06.06.2020). 
durch reduzierte Absatzchancen am Markt, das Ausbleiben notwendiger Zulieferungen oder das krankheitsbedingte Fehlen einer für den ertragreichen Betrieb erforderlichen Belegschaftsgröße beeinträchtigt werden. Umgekehrt ist aber auch denkbar, dass das Leistungsinteresse des Arbeitgebers pandemiebedingt wächst: Besonders die systemrelevanten Einrichtungen, aber auch Unternehmen der Lebensmittel- und Logistikbranche stoßen in der Pandemie an ihre Kapazitätsgrenzen, die durch Krankheitsausfälle und mitunter steigende Nachfrage in einzelnen Marktsegmenten vor zusätzliche Belastungsproben gestellt werden.

Aber auch, wenn der Arbeitnehmer zur Arbeit fähig und bereit ist und der Arbeitgeber weiterhin ein Interesse am Erhalt der Arbeitsleistung hat, bestehen in der Coronakrise besondere Gefahren für das Integritätsinteresse der Parteien, welche durch das Vertragsrecht abgebildet werden müssen. So wird der Arbeitgeber die durch das Infektionsrisiko erhöhte Erkrankungsgefahr durch geeignete Schutzmaßnahmen reduzieren müssen, um dem Risiko einer Ausbreitung der Krankheit in der Belegschaft entgegenzutreten. Will er eine Kettenansteckung verhindern, wird er dabei ein Interesse an Aufklärung über personenbezogene Krankheitsinformationen haben, um potenzielle Ansteckungsherde frühzeitig zu erkennen und zu isolieren. Dies wirft neben datenschutzrechtlichen Fragen auch Haftungsfragen auf, wenn der Arbeitgeber oder infizierte Arbeitnehmer die zur Verhinderung einer Ausbreitung der Infektion erforderlichen Maßnahmen unterlassen und hierdurch die Gesundheit anderer Arbeitnehmer gefährden.

Diese drei Problemkreise sollen im Folgenden schrittweise auf ihre Behandlung durch das Arbeitsvertragsrecht untersucht werden. Dabei soll der Identifikation normativer Grundentscheidungen der arbeitsrechtlichen Risikotragung Vorrang vor der Beantwortung spezieller Rechtsfragen eingeräumt werden, um der eingangs beschriebenen Diffusion einzelfallabhängiger Subsumtionsergebnisse, die in Anbetracht der Diversität von Arbeitsverhältnissen unausweichlich ist, entgegenzuwirken.

\section{B. Pandemiebedingte Arbeitsunfähigkeit/-unwilligkeit des Arbeitnehmers}

Risiken für den Leistungsaustausch im Arbeitsverhältnis können sich in der Coronakrise zunächst daraus ergeben, dass der Arbeitnehmer zur Arbeitsleistung unfähig oder unwillig ist. Dabei sind Fälle, in denen sich die Unfähigkeit bzw. Unwilligkeit des Arbeitnehmers auf die gesamte Arbeitsleistung bezieht, von solchen zu unterscheiden, in denen er lediglich zur Durchführung einzelner Weisungen außer Stande oder nicht bereit ist. In 
all diesen Fällen liegt eine Leistungsstörung aus der Risikosphäre des Arbeitnehmers vor, deren Realisierungsfolgen grundsätzlich auch von diesem zu tragen wären. Von dem Grundsatz „Ohne Arbeit, kein Lohn“ machen jedoch sowohl das Allgemeine Schuldrecht als auch das Arbeitsrecht zahlreiche Ausnahmen zugunsten des Arbeitnehmers, die in der Coronakrise sein Vertrauen auf Erhalt seines Arbeitsentgelts besonders schützen.

\section{Wegfall oder Beschränkung der Leistungsfähigkeit}

Für den Fall, dass der Arbeitnehmer selbst am Coronavirus erkrankt, wird er gem. $\$ 275$ I BGB von der Leistungspflicht befreit. ${ }^{4} \mathrm{Ihm}$ steht, wie jedem krankheitsbedingt arbeitsunfähigen Arbeitnehmer, ein Anspruch auf Entgeltfortzahlung nach $\mathbb{3}$ I EFZG zu. ${ }^{5}$ Dieser ist ausgeschlossen, wenn der Arbeitnehmer schuldhaft erkrankt ist, was insbesondere dann der Fall sein kann, wenn er grob gegen innerbetriebliche Schutzmaßnahmen verstoßen oder sich entgegen behördlicher Warnungen in ein Gefahrengebiet begeben hat. ${ }^{6}$ Die für den Nachweis der Erkrankung erforderliche Arbeitsunfähigkeitsbescheinigung kann während der Kernzeit der Pandemie ausnahmsweise auch nach telefonischer Anamnese für bis zu zwei Wochen ausgestellt werden, ohne dass es eines analogen Arztbesuches bedarf. ${ }^{7}$

Besteht lediglich der Verdacht einer Coronainfektion, z.B. weil der Arbeitnehmer Kontakt zu positiv getesteten Personen hatte, so wird er hierdurch allein nicht arbeitsunfähig, da die Arbeitsleistung grundsätzlich

4 Allgemein zu den Folgen einer Krankheit für die Arbeitsleistungspflicht ErfK/B. Reinhard, 20. Auflage 2020, 33 EFZG Rn. 3; U. Preis, Individualarbeitsrecht, 5. Auflage 2017, Rn. 2021; speziell zur Corona-Erkrankung A. Sagan/M. Brockfeld, NJW 2020, 1112, 1113; A. Groeger, ARP 2020, 106, 108; differenzierender F. J. Düwell, der in einem symptomfreien Krankheitsverlauf keine Einschränkung der Arbeitsfähigkeit sehen will, BB 2020, 891, 892.

5 ErfK/B. Reinhard, $\$ 3$ EFZG Rn. 45; U. Preis, Individualarbeitsrecht, Rn. 2172.

6 A. Sagan/M. Brockfeld, NJW 2020, 1112, 1113; E. Dehmel/N. Hartmann, BB 2020, 885, 888; M. Fublrott/K. Fischer wollen ein solches Verschulden nur im Einzelfall annehmen, wenn das Infektionsrisiko im Gefahrengebiet höher ist als in Deutschland, da sich sonst lediglich das (derzeit gesteigerte) allgemeine Lebensrisiko verwirklicht, NZA 2020, 345, 347.

7 Vgl. $₫ 31$ S. 3 und 4 des Bundesmantelvertrages-Ärzte, welcher am 11.03.2020 durch Zusatzvereinbarung zunächst für einen befristeten Zeitraum von vier Wochen dahingehend angepasst wurde, dass eine telefonische Anamnese bei Erkrankungen der oberen Atemwege zur Feststellung der Arbeitsunfähigkeit genügen soll, vertiefend: F. J. Düwell, BB 2020, 891, $893 \mathrm{f}$. 
möglich bleibt. Wird er infolge des Kontaktes gem. $\$ 30$ IfSG unter Quarantäne gestellt, wird ihm die Arbeitsleistung regelmäßig rechtlich unmöglich sein; dies gilt jedenfalls dann, wenn die zuständige Behörde zugleich ein berufliches Tätigkeitsverbot nach $\$ 31$ IfSG ausspricht. ${ }^{8}$ In diesen Fällen wird dem Arbeitnehmer regelmäßig $\$ 616$ BGB zugutekommen, welcher seinen Entgeltanspruch erhält, wenn er für eine verhältnismäßig nicht erhebliche Zeit durch einen in seiner Person liegenden Grund ohne sein Verschulden an der Leistung verhindert wird. ${ }^{9}$ Was dabei eine „verhältnismäßig nicht erhebliche Zeit" sein soll, ist nach den Umständen der konkreten Verhinderung zu bestimmen, wobei sich im Falle der behördlich angeordneten Isolation in Anbetracht der Vergleichbarkeit mit dem tatsächlichen Erkrankungsfall eine Orientierung an der 6-Wochen-Frist des EFZG anbietet. ${ }^{10} \mathrm{Im}$ Falle eines behördlichen Tätigkeitsverbotes steht dem Arbeitnehmer ein Entschädigungsanspruch gem. $\$ 56$ IfSG zu, welcher in den ersten sechs Wochen vom Arbeitgeber auszuzahlen ist und diesem von der Behörde erstattet wird. Aus praktischer Sicht ist dieser Anspruch insofern wichtig, da $\$ 616$ BGB als disponibles Vertragsrecht in Arbeitsverträgen nicht selten abbedungen ist. ${ }^{11}$

Die Leistungsfähigkeit des Arbeitnehmers kann in der Coronakrise aber nicht nur durch die eigene Infektion oder einen entsprechenden Verdacht beeinträchtigt sein. Infolge der Schließung sämtlicher Schulen und Kitas stehen viele Arbeitnehmer vor der Notwendigkeit, während der täglichen Arbeitszeit ihren Betreuungspflichten nachzukommen; gleiches gilt freilich erst recht, wenn das betreuungsbedürftige Kind selbst erkrankt. In beiden Fällen kann der Arbeitnehmer die Leistung gem. $\$ 275$ III BGB wegen Unzumutbarkeit verweigern, was allerdings grundsätzlich gem. \$326 I BGB auch den Untergang seines Vergütungsanspruches zur Folge hat. ${ }^{12}$ $\$ 616$ BGB wird dem Arbeitnehmer - soweit nicht ohnehin abbedungen regelmäßig nur bei einer Erkrankung des Kindes helfen, da die wochenlangen Schul- und Kitaschließungen den Tatbestand regelmäßig nicht mehr

8 K.-S. Hohenstatt/C. Krois, NZA 2020, 413, 414; A. Sagan/M. Brockfeld, NJW 2020, 1112, 1113.

9 A. Sagan/M. Brockfeld, NJW 2020, 1112, 1113.

10 ErfK/U. Preis, $\mathbb{S} 616$ BGB Rn. 10 a; kritisch A. Sagan/M. Brockfeld, NJW 2020, 1112, 1113.

11 M. Fublrott, GWR 2020, 107, 108; J. Grüneberg, ARP 2020, 111, 115; K.-S. Hohenstatt/C. Krois, NZA 2020, 413, 416.

12 A. Sagan/M. Brockfeld, NJW 2020, 1112, 1114; M. Fublrott, GWR 2020, 107, 108; E. Dehmel/N. Hartmann, BB 2020, 885, 886. 
erfüllen werden. ${ }^{13}$ Dieser Lücke hat der Gesetzgeber allerdings zuletzt durch Schaffung des $\$ 56$ Ia IfSG Abhilfe geleistet, indem er einen Entschädigungsanspruch für Sorgeberechtigte von Kindern, die das zwölfte Lebensjahr noch nicht vollendet haben, für Verdienstausfälle in das Infektionsschutzgesetz implementierte.

\section{Generelle Arbeitsunwilligkeit des Arbeitnehmers}

Ein generelles Leistungsverweigerungsrecht steht dem Arbeitnehmer auch in der Coronakrise grundsätzlich nicht zu. Die bloße Befürchtung einer Infektion im Betrieb des Arbeitgebers begründet ein solches nicht. ${ }^{14}$ Im Einzelfall - man denke hier insbesondere an Risikogruppen und schwangere Arbeitnehmerinnen, bei denen die Gefahr eines lebensbedrohlichen Krankheitsverlaufes größer oder die zu befürchtenden Folgen eines solchen Verlaufs schwerwiegender ist - wird man jedoch ein Recht zu Leistungsverweigerung nach $\mathbb{2} 275$ III BGB jedenfalls dann erwägen müssen, wenn eine alternative Beschäftigung am Wohnsitz des Arbeitnehmers nicht möglich ist. ${ }^{15}$

Der Arbeitnehmer kann seine Arbeitsleistung jedoch gem. \$273 I BGB zurückbehalten, solange der Arbeitgeber seinen arbeitsvertraglichen Schutzpflichten gem. $\$ 618$ BGB nicht hinreichend nachkommt. ${ }^{16}$ Dann behält er gem. $\$ 615$ S. 1 BGB sogar seinen Anspruch auf die Vergütung, da sich der Arbeitgeber durch die fehlende Bereitstellung eines zumutbaren Arbeitsplatzes in Annahmeverzug befindet. ${ }^{17}$

13 A. Sagan/M. Brockfeld, NJW 2020, 1112, 1114; E. Dehmel/N. Hartmann, BB 2020, 885, 886 f.; K.-S. Hohenstatt/C. Krois, NZA 2020, 413, 416; offener M. Fuhlrott/K. Fischer, NZA 2020, 345, 348, die den Vergütungsanspruch allerdings nur für einen Zeitraum von ca. fünf Arbeitstagen erhalten wollen, innerhalb derer die Organisation einer alternativen Kinderbetreuung möglich sein solle.

14 M. Fublrott, GWR 2020, 107; E. Dehmel/N. Hartmann, BB 2020, 885, 885 f.; A. Groeger, ARP 2020, 106.

15 M. Fublrott, GWR 2020, 107, 108; E. Dehmel/N. Hartmann, BB 2020, 885, 886.

16 A. Sagan/M. Brockfeld, NJW 2020, 1112, 1114; zum Umfang dieser Pflichten sogleich unter IV.

17 ErfK/R. Wank, $\$ 618$ BGB Rn. 27 m.w.N. 


\section{Weigerung zur Durchfübrung einzelner Weisungen}

Regelmäßig wird der Arbeitnehmer die Arbeitsleistung ohnehin nicht gänzlich verweigern, sondern sich lediglich einzelner Weisungen unter Verweis auf die bestehende Pandemie entziehen wollen. Im Hinblick auf die geltenden Mobilitätseinschränkungen können Arbeitnehmer vor allem gewillt sein, ihre Arbeitsleistung am Wohnsitz zu erbringen, um dem Risiko einer Infektion im Betrieb zu entgehen. Ein allgemeiner Anspruch des Arbeitnehmers auf Homeoffice besteht allerdings auch während der Coronapandemie nicht. ${ }^{18}$ Ein solcher kann sich aber ausnahmsweise aus arbeitsvertraglichen Schutzpflichten ergeben, wenn einem Arbeitnehmer mit höherem Infektionsrisiko, der seine Arbeitsleistung ohne erhebliche Einschränkungen von zu Hause erbringen kann, der Weg zu seinem Arbeitsplatz nicht zugemutet werden kann. ${ }^{19}$ Lässt der Arbeitgeber in der Pandemie einen Teil der Belegschaft aus dem Homeoffice arbeiten, kann sich für den übrigen Teil ein Anspruch aus dem arbeitsrechtlichen Gleichbehandlungsgrundsatz ergeben, soweit eine Ungleichbehandlung einzelner Arbeitnehmer nicht gerechtfertigt werden kann. ${ }^{20}$

Umgekehrt stellt sich die Frage, ob die einseitige Anordnung der Durchführung von Homeoffice-Arbeiten durch den Arbeitgeber von dessen Weisungsrecht nach $\$ 106$ S. 1 GewO gedeckt ist. Eine entsprechende Weisung wird sich regelmäßig wohl in den Grenzen des billigen Ermessens bewegen, berücksichtigt man, dass es sich hierbei um eine vom RKI empfohlene Maßnahme zur Reduktion der Infektionsrisiken handelt, die gerade auch dem Schutz der Gesundheit des angewiesenen Arbeitnehmers selbst dient. ${ }^{21}$ Freilich hat der Arbeitgeber dem Arbeitnehmer Betriebsmit-

18 E. Dehmel/N. Hartmann, BB 2020, 885, 886; A. Sagan/M. Brockfeld, NJW 2020, 1112, 1115; allgemein zu einem Anspruch des Arbeitnehmers auf häusliches Arbeiten Picker, ZfA 2019, 269.

19 E. Dehmel/N. Hartmann, BB 2020, 885, 886; A. Sagan/M. Brockfeld, NJW 2020, $1112,1115$.

20 A. Sagan/M. Brockfeld, NJW 2020, 1112, 1115.

21 A. Sagan/M. Brockfeld, NJW 2020, 1112, 1114; M Fublrott/K. Fischer, NZA 2020, 345, 349 f.; S. Krieger/T. Rudnik/A. Povedano Peramato, NZA 2020, 473, 475; differenzierend E. Dehmel/N. Hartmann, die unter Verweis auf Art. 13 GG eine Pflicht zur Arbeit im Homeoffice und zur Duldung der Einrichtung eines heimischen Arbeitsplatzes grundsätzlich ablehnen, die Anordnung der Arbeit im Mobile-Office aber im Pandemiefall für zulässig erachten, BB 2020, 885, 886, 888; a.A. A. Groeger, ARP 2020, 106, 107, unter Verweis auf LAG Berlin-Brandenburg, Urt. v. 14.11.2018 - 17 Sa 562/18; M. Weber, ARP 2020, 120, 122. 
tel für die heimische Tätigkeit bereitzustellen und auch im Homeoffice arbeitsschutzrechtliche Vorgaben zu erfüllen. ${ }^{22}$

Auch Weisungen zur Durchführung von Dienstreisen oder Tätigkeiten mit erhöhtem Infektionsrisiko sind in der Pandemie im Ausgangspunkt als billig zu erachten und können vom Arbeitnehmer nicht ohne Weiteres missachtet werden. Vielmehr muss jeweils im Einzelfall erwogen werden, ob eine Weisung aufgrund der jeweils aktuellen Infektionslage ausnahmsweise als unbillig einzustufen ist, wobei insbesondere die körperliche Verfassung des betroffenen Arbeitnehmers und das Interesse des Arbeitgebers an der Durchführung der Weisung zu berücksichtigen sind. ${ }^{23}$

\section{Pandemiebedingte Schwankungen des Leistungsinteresses des Arbeitgebers}

Auf Seiten des Arbeitgebers droht die Pandemie vor allem, Einfluss auf sein Interesse am Erhalt der Arbeitsleistung zu nehmen. So mussten infolge der bundesweiten Kontaktbeschränkungen zahlreiche Arbeitgeber insbesondere im Dienstleistungssektor ihren Betrieb vorübergehend einstellen, weshalb sie für diesen Zeitraum ihr Interesse an Arbeitsleistung weitgehend verloren haben. In vielen Branchen ist zudem die Nachfrage bedingt durch die wirtschaftlichen Unwägbarkeiten der Krise teils dramatisch eingebrochen, wodurch viele Arbeitgeber selbst bei laufendem Betrieb ihre Belegschaft nicht vollständig auslasten können. In anderen Branchen wiederum - allen voran im Gesundheitssektor und in der Logistikbranche - haben Arbeitgeber umgekehrt mit Arbeitskräftemangel zu kämpfen, soweit ihre Leistungen in der Pandemie am Markt besonders nachgefragt werden und die vorhandene Personaldecke durch Häufung von Verdachts- und Erkrankungsfällen abzuschmelzen droht. Dabei handelt es sich um Verwendungsrisiken, die das Nutzungsinteresse des Arbeitgebers betreffen und die daher im Ausgangspunkt seiner Sphäre zuzurechnen sind.

22 A. Sagan/M. Brockfeld, NJW 2020, 1112, 1114; S. Krieger/T. Rudnik/A. Povedano Peramato, NZA 2020, 473, 479.

23 In Bezug auf die Weisung zur Durchführung von Dienstreisen Fublrott, GWR 2020, 107, 108; A. Sagan/M. Brockfeld, NJW 2020, 1112, 1115. 


\section{Ausfall oder Beschränkung des Leistungsinteresses}

Den Ausfall des Leistungsinteresses regelt das Arbeitsrecht in $₫ 615$ BGB, nach dessen Satz 1 der Arbeitgeber im Falle seines Annahmeverzuges Vergütung auch für solche Leistungen schuldet, die der Arbeitnehmer in der Folge nicht erbringen konnte, ohne dass dieser zur Nachleistung verpflichtet ist. Damit wird dem Arbeitgeber das Wirtschaftsrisiko des Arbeitsverhältnisses auferlegt; ist die Fortsetzung seines Betriebes infolge der Coronakrise wegen Auftrags- oder Absatzmangels wirtschaftlich sinnlos, hat er seinen Arbeitnehmern dennoch die vertragliche Vergütung zu zahlen. ${ }^{24}$

\section{Die arbeitsvertragliche Betriebsrisikolehre}

In seinem Satz 3 erweitert $\$ 615$ BGB diesen Grundsatz, indem er dem Arbeitgeber auch das sog. Betriebsrisiko zuweist, also das Risiko, die Belegschaft auch ohne eigenes Verschulden aus betriebstechnischen Gründen nicht beschäftigen zu können. ${ }^{25}$ Davon erfasst sind solche Risiken, die sich aus dem Betrieb oder seiner Belegenheit ergeben, also z.B. Rohstoff- oder Arbeitskraftmangel, Betriebsmittelausfälle oder Naturkatastrophen. ${ }^{26}$

$\mathrm{Ob}$ vom Betriebsrisiko auch eine Betriebsuntersagung durch die bundesweit eingeführten Eindämmungsverordnungen erfasst ist, ist freilich nicht abschließend geklärt. Im Grundsatz hat der Arbeitgeber solche Untersagungsrisiken zu tragen, die durch die besondere Eigenart seines Betriebes bedingt sind. ${ }^{27}$ Vor dem Hintergrund der Reichweite der Schließungsverordnungen wird daher vertreten, dass es sich hierbei nicht um ein betriebsbezogenes Ausfallrisiko handele, sondern sich in der Pandemie lediglich das allgemeine Lebensrisiko verwirkliche, welches der Ar-

24 ErfK/U. Preis, $\$ 615$ Rn. 120 f.; MüKo-BGB/M. Henssler, 8. Auflage 2020, $\$ 615$ Rn. 98.

25 Freilich lässt sich zweifeln, ob es für diese Risikozuweisung tatsächlich eines dritten Satzes bedurfte, wie er durch die Schuldrechtsmodernisierung zur Kodifizierung der Betriebsrisikolehre des BAG Einzug in das BGB fand, vgl. Kritik bei Staudinger/R. Richardi/P. Fischinger, 2016, \$ 615 Rn. $210 \mathrm{ff}$.

26 ErfK/U. Preis, $\$ 615$ Rn. 130 f.; MüKo-BGB/M. Henssler, $\$ 615$ Rn. 96.

27 A. Sagan/M. Brockfeld, NJW 2020, 1112, 1116; ErfK/U. Preis, \$615 Rn. 132; Staudinger/R. Richardi/P. Fischinger, $\$ 615$ Rn. 212; a.A. K.-S. Hohenstatt/C. Krois, NZA 2020, $413 \mathrm{f}$., wenn sie die Möglichkeit zur anderweitigen Erbringung der vereinbarten Dienste zum zentralen Abgrenzungskriterium erheben, welches bei allgemeinen, tätigkeitsbezogenen Betriebsuntersagungen trotz ihrer Betriebszugehörigkeit gerade nicht zu einer Lohnfortzahlungspflicht des Arbeitgebers führt. 
beitgeber nicht abstrakt beherrschen könne. ${ }^{28}$ Dagegen lässt sich allerdings einwenden, dass die jeweiligen Verordnungen die Betriebsuntersagung wenngleich in der Summe weitreichend - doch in aller Regel in Bezug auf ihren Geschäftsgegenstand anordnen. So regelt beispielsweise die Verordnung zur Eindämmung des Coronavirus in Berlin in den $\mathbb{\$} \$ 2-13$ verschiedenste Gruppen von Gewerbebetrieben und differenziert innerhalb dieser zwischen Betrieben, die nicht für den Publikumsverkehr zu öffnen sind und solchen, die aufgrund ihrer Bedeutung für das öffentliche Leben trotz Pandemie weiter betrieben werden können. Insofern besteht im Pandemiefall sogar ein engerer Bezug zwischen Betriebsausfall und dem Geschäftsgegenstand des Arbeitgebers, als dies regelmäßig bei Naturkatastrophen der Fall sein wird. ${ }^{29}$ Dieses Ergebnis erscheint auch interessengerecht, berücksichtigt man, dass der Arbeitnehmer das Pandemierisiko seinerseits genauso wenig beherrschen kann wie der Arbeitgeber und das wirtschaftliche Risiko bei Unanwendbarkeit des $\$ 615$ S. 3 BGB vollständig allein zu tragen hätte, obwohl er selbst zur Leistung bereit und imstande ist.

Der Arbeitgeber wird hierdurch auch nicht unbillig belastet. Zum einen kann er die Verwirklichungsfolgen - ähnlich wie Naturkatastrophen - jedenfalls insoweit abstrakt beherrschen, dass er sich gegen pandemiebedingte Betriebsausfälle versichern kann. ${ }^{30}$ Führt die Lohnfortzahlung nach vorübergehender Betriebsschließung dennoch zu einer wirtschaftlichen Existenzgefährdung des Betriebes, so soll die Lohnfortzahlungspflicht nach Rechtsprechung des BAG zudem ausnahmsweise entfallen, ${ }^{31}$ wobei zu erwarten ist, dass das BAG diese Rechtsprechung auf die hier gegenständli-

28 A. Sagan/M. Brockfeld, NJW 2020, 1112, 1116 unter Verweis auf Koller, der die abstrakte Beherrschbarkeit als maßgebliches Abgrenzungsmerkmal arbeitsvertraglicher Betriebsrisikoverteilung ansieht, in: Die Risikozurechnung bei Vertragsstörungen im Austauschverhältnis, 1979, 390 ff., 423 ff.; ähnlich E. Dehmel/N. Hartmann, BB 2020, 885, 891, die eine solche Risikoveranlagung allenfalls bei Betrieben im Lebensmittel- und/oder Medizinsektor anerkennen wollen; a.A. M. Fublrott/K. Fischer, die auch bei behördlicher Schließung generell einen Fall von $\$ 615$ BGB annehmen, NZA 2020, 345, 348.

29 Beispielhaft BAGE 42, 94, wonach der Arbeitgeber das Risiko eines Ausfalls der Heizungsanlagen wegen eines plötzlichen Kälteeinbruchs zu tragen hat, ohne dass es auf die besonderen Eigenarten des Betriebes ankommt.

30 Spätestens mit der Coronakrise dürften Pandemiefälle in der Versicherungspraxis als ein zu berücksichtigender Risikofaktor für Unternehmen anzusehen sein, vgl. zum Versicherungsschutz im Pandemiefall V. Schreier, VersR 2020, 513.

31 BAG AP BGB $\$ 615$ Betriebsrisiko Nr. 28, aber kritisch: A. Sagan/M. Brockfeld, NJW 2020, 1112, 1116. 
chen Fälle übertragen wird. ${ }^{32}$ Insofern besteht gar keine Notwendigkeit, den Arbeitgeber vom pandemiebedingten Betriebsuntersagungsrisiko zu Lasten seiner Arbeitnehmer zu befreien, zumal die Frage nach Entschädigungsansprüchen der Betriebsinhaber gegen den Staat bislang gerichtlich nicht geklärt ist. ${ }^{33}$

\section{Reaktionsmöglichkeiten des Arbeitgebers}

Muss der Arbeitgeber danach seine Arbeitnehmer auch für den Fall entlohnen, dass er ihre Arbeitsleistung nicht verwenden kann, wird er regelmäßig ein Interesse daran haben, sich von einzelnen Arbeitnehmern zu trennen, um in der Krise Lohnkosten einzusparen. Die betriebsbedingte Kündigung kann dabei allerdings nur ultima ratio sein. ${ }^{34}$ Daran sind im Pandemiefall insoweit hohe Anforderungen zu stellen, da der Arbeitsmangel hier oftmals nur vorübergehender Natur ist, was für eine betriebsbedingte Kündigung regelmäßig unzureichend sein wird. ${ }^{35}$ Erforderlich für eine Kündigung aus dringenden, betrieblichen Erfordernissen wird daher eine Prognose sein, dass der Personalbedarf zumindest auch in der Folgezeit der Pandemie aufgrund der zu erwartenden, volkswirtschaftlichen Schäden hinter dem Vorkrisenniveau zurückbleibt. ${ }^{36}$

Bevor der Arbeitgeber allerdings betriebsbedingte Kündigungen überhaupt aussprechen kann, hat er zunächst sämtliche Handlungsalternativen $\mathrm{zu}$ erwägen, um eine einseitige Vertragsbeendigung zu vermeiden. So kann der Arbeitsmangel teilweise durch den Abbau von Überstunden- und Arbeitszeitkonten kompensiert werden. ${ }^{37}$ Der Arbeitgeber kann Arbeitnehmer weiterhin freistellen, wobei eine unentgeltliche Freistellung nur in Einvernehmen mit dem Arbeitnehmer erfolgen kann, da der Vergütungsanspruch bei einseitiger Freistellung in der Regel gem. $\$ 615$ S. 1 BGB we-

32 M. Fublrott/K. Fischer, NZA 2020, 345, 348; M. Fublrott, GWR 2020, 107, 110.

33 Entschädigungsansprüche befürwortend Antweiler, NVwZ 2020, 584, 589; M-P. Weller/M. Lieberknecht/V. Habrich, NJW 2020, 1017, 1019.

34 Allgemein zum Ultima-Ratio-Prinzip der betriebsbedingten Kündigung U. Preis, Individualarbeitsrecht, Rn. $2856 \mathrm{ff}$.

35 C. Zieglmeier, DStR 2020, 729, 734; insbesondere die Einführung von Kurzarbeit wird regelmäßig für einen nur vorübergehenden Arbeitsmangel sprechen, vgl. BAG NZA 2012, 852, $854 \mathrm{f}$.

36 C. Zieglmeier, DStR 2020, 729, 734.

37 A. Sagan/M. Brockfeld, NJW 2020, 1112, 1115. 
gen Nichtannahme der Arbeitsleistung erhalten bleibt. ${ }^{38}$ Eine Überbrückung der Krise ist grundsätzlich auch durch Urlaub möglich, wobei der Arbeitgeber auch diesen nicht einseitig anordnen kann. Eine Ausnahme bildet insoweit die Anordnung von Betriebsferien, die aber aufgrund der langen Vorankündigungsfristen des $\$ 7$ I BUrlG in der Coronakrise zur Bewältigung der Unterbeschäftigung ungeeignet erscheint. ${ }^{39}$

Als praxisrelevanteste Möglichkeit zur Reduzierung der Lohnkosten steht dem Arbeitgeber zudem die Möglichkeit zur Einführung von Kurzarbeit nach Maßgabe der $\$ \$ \$ 95 \mathrm{ff}$. SGB III offen. Diese kann auf Grundlage einer Betriebsvereinbarung sogar ohne unmittelbare Einwilligung der betroffenen Arbeitnehmer erfolgen. ${ }^{40}$ Zentrale Voraussetzung hierfür ist, dass ein erheblicher, vorübergehender und nicht vermeidbarer Arbeitsausfall droht. Mit dem „Gesetz zur befristeten krisenbedingten Verbesserung der Regelungen für das Kurzarbeitergeld" ${ }^{41}$ hat der Gesetzgeber die Anforderungen hieran deutlich erleichtert, so dass von einem erheblichen Arbeitsausfall bereits dann auszugehen ist, wenn $10 \%$ der in einem Betrieb beschäftigten Arbeitnehmer vom Ausfall betroffen sind. In der Praxis zeichnet sich bereits $a b$, dass viele Unternehmen von dieser Möglichkeit Gebrauch machen, um die eigenen Zahlungspflichten in der Krise zu reduzieren, ohne die Personaldecke besonders mit Blick auf die Nachkrisenzeit zu reduzieren.

\section{Steigerung des Leistungsinteresses}

Vereinzelt ist aber auch zu beobachten, dass Arbeitgeber während der Pandemie einen Mehrbedarf an Arbeitsleistung haben. Kurzfristig lässt sich dieser durch Anordnung von Überstunden oder Einführung weiterer Arbeitsschichten befriedigen, wobei natürlich die arbeitszeitrechtlichen Vorgaben einzuhalten und insbesondere Ausgleichszeiträume vorzuhalten sind. ${ }^{42}$ Gem. $\$ 15$ II ArbZG kann der Arbeitgeber auf Antrag eine behördli-

38 Erfolgt die Freistellung wegen Krankheitsverdachts, wird der Entgeltanspruch regelmäßig gem. $\$ 616$ BGB erhalten bleiben, A. Sagan/M. Brockfeld, NJW 2020, 1112, 1114.

39 A. Sagan/M. Brockfeld, NJW 2020, 1112, 1115; M. Fublrott/K. Fischer, NZA 2020, 345, 348; M. Fublrott, GWR 2020, 107, 110.

40 M. Fublrott/K. Fischer, NZA 2020, 345, 346; M. Fublrott, GWR 2020, 107, 110.

41 BGBl. I 2020, S. 493.

42 M. Fublrott, GWR 2020, 107, 109; mit $\$ 14$ IV ArbZG hat der Gesetzgeber insoweit eine neue Verordnungsermächtigung im Pandemiefall für solche Tätigkeiten 
che Genehmigung zur Sonn- und Feiertagsarbeit erhalten, wenn diese im öffentlichen Interesse dringend nötig wird. Diese Möglichkeit ist besonders für solche Unternehmen relevant, die in der gegenwärtigen Krise mit der Produktion wichtiger medizinischer Güter wie Testsubstanzen, Desinfektionsmittel oder Schutzkleidung betraut sind. ${ }^{43}$ Reicht das vorhandene Personal zur Deckung des Arbeitskraftbedarfs während der Pandemie nicht aus, kann der Arbeitgeber auch befristete Neueinstellungen vornehmen, die gem. $\$ 14$ II TzBfG für eine Dauer von zwei Jahren sachgrundlos erfolgen können; denkbar ist zudem eine Sachgrundbefristung wegen vorübergehendem betrieblichem Bedarf ( $\$ 14$ I 2 Nr. 1 TzBfG) oder zur Vertretung erkrankter Arbeitnehmer (\$14 I 2 Nr. 3 TzBfG). ${ }^{44}$

\section{Besondere Vertragsdurchführungsrisiken}

Doch auch wenn das Arbeitsverhältnis in der Coronakrise grundsätzlich weiter durchgeführt werden kann, ergeben sich aus der gegenwärtigen Infektionslage besondere Vertragsrisiken, die insbesondere auf die im Vergleich zur häuslichen Isolation erhöhte Infektionsgefahr der Arbeitnehmer im Betrieb des Arbeitgebers zurückzuführen sind. Aufgabe des Arbeitsvertragsrechts ist es hier, durch eine Konkretisierung arbeitsvertraglicher Schutz- und Treuepflichten das Infektionsrisiko sämtlicher Arbeitnehmer so weitgehend wie möglich zu reduzieren. Die Eindämmung betrieblicher Infektionsherde erfolgt im durchgeführten Arbeitsverhältnis insbesondere durch die Begründung besonderer Schutz- und Aufklärungspflichten.

\section{Schutzpflichten des Arbeitgebers}

Gem. $\$ 3$ ArbSchG ist der Arbeitgeber verpflichtet, die erforderlichen Maßnahmen des Arbeitsschutzes unter Berücksichtigung der Umstände zu treffen, die Sicherheit und Gesundheit der Beschäftigten auf der Arbeit beein-

eingeführt, die die öffentliche Sicherheit und Ordnung, das Gesundheitswesen, die pflegerische Versorgung, die Daseinsvorsorge sowie die Versorgung der Bürger mit existenziellen Gütern aufrechterhalten.

43 M. Fublrott/K. Fischer, NZA 2020, 345, 346 f.; M. Fublrott, GWR 2020, 107, 109; für private Paketzusteller hat das VG Berlin ein erforderliches öffentliches Interesse in Ermangelung einer allgemeinen Versorgungskrise im Eilrechtsschutz aber bereits abgelehnt, Beschl. v. 09.04.2020 - 4 L 132/20 (u.a.).

44 A. Sagan/M. Brockfeld, NJW 2020, 1112, 1115. 
flussen. Über $\$ 618$ I BGB finden diese Schutzpflichten Einzug in die schuldrechtliche Beziehung der Parteien und begründen einen Anspruch des Arbeitnehmers gegen den Arbeitgeber, ihm zumutbare Maßnahmen zu ergreifen, um Arbeitnehmer möglichst effektiv vor einer Infektion zu schützen. ${ }^{45}$ Die Beurteilung der erforderlichen Maßnahmen hat dabei die sich verändernde Infektionslage zu berücksichtigen ( $\$ 3$ I 2 ArbSchG) und hat arbeitsplatzbezogen zu erfolgen. ${ }^{46} \mathrm{Zu}$ den erforderlichen Maßnahmen werden dabei zunächst solche zu zählen sein, die während der Pandemie auch für den gemeinsamen Umgang im öffentlichen Raum gelten (Abstandsgebote, Hygieneanforderungen, Meidung persönlicher Kontakte). ${ }^{47}$ Daneben kommen je nach Eigenart des Arbeitsplatzes auch die Errichtung physischer Barrieren, die Verkürzung von Reinigungsintervallen und die Verwendung angemessener Schutzkleidung in Betracht. ${ }^{48}$ Gem. $\$ 4$ Nr. 6 ArbSchG hat der Arbeitgeber die Bedürfnisse von Beschäftigtengruppen mit erhöhtem Infektionsrisiko besonders zu berücksichtigen. ${ }^{49}$ Eine Nichterfüllung dieser Schutzpflichten erlaubt dem Arbeitnehmer die Leistungsverweigerung gem. $\$ 273$ I BGB, wobei sein Entgeltanspruch nach $\$ 615$ S. 1 BGB fortbesteht. ${ }^{50}$

\section{Aufklärungspflichten}

$\mathrm{Zu}$ einer effektiven Infektionseindämmung wird der Arbeitgeber regelmäBig aber nur in der Lage sein, wenn er über eine hinreichende Informationsgrundlage über den Gesundheitszustand seiner Arbeitnehmer verfügt. Nur so ist es ihm möglich, potenzielle Infektionsherde frühzeitig zu erkennen und zu isolieren, um eine Ausbreitung der Infektion in der Belegschaft zu vermeiden. Umgekehrt werden aber auch Arbeitnehmer in der Coronakrise ein Interesse daran haben, zu erfahren, von welchen Kolleginnen und Kollegen die Gefahr einer Ansteckung ausgeht. Das Arbeitsver-

45 ErfK/R. Wank, $\$ 618$ BGB Rn. 4; U. Preis, Individualarbeitsrecht, Rn. 1795; MüKoBGB/M. Henssler, $\$ 618$ Rn. 9.

46 A. Groeger, ARP 2020, 106, 110; A. Sagan/M. Brockfeld, NJW 2020, 1112, 1113.

47 A. Sagan/M. Brockfeld, NJW 2020, 1112, 1113; M. Weber will indes Pflichten zur Hygienevorkehrung erst erwachsen lassen, wenn die abstrakte Gefährdungslage im Betrieb in eine konkrete Infektionsgefahr umgeschlagen ist, ARP 2020, 120, 121.

48 A. Sagan/M. Brockfeld, NJW 2020, 1112, 1113.

49 A. Sagan/M. Brockfeld, NJW 2020, 1112, 1113.

50 ErfK/R. Wank, \618 BGB Rn. 25, 27; U. Preis, Individualarbeitsrecht, Rn. 1795; MüKo-BGB/M. Henssler, $\mathbb{} 618 \mathrm{Rn} .92 \mathrm{f}$. 
tragsrecht hat hier durch Konkretisierung der arbeitsvertraglichen Aufklärungspflichten die Informationsinteressen der Parteien mit dem Persönlichkeitsschutz der Arbeitnehmer in Einklang zu bringen.

\section{Aufklärungspflichten des Arbeitnehmers}

Grundsätzlich gilt für das Arbeitsrecht, dass der Arbeitnehmer gegenüber dem Arbeitgeber nicht zur Aufdeckung eigener Krankheitsbilder verpflichtet ist. Sein allgemeines Persönlichkeitsrecht geht dem Informationsinteresse des Arbeitgebers vor, da diesem mit der Erbringung eines Nachweises über die krankheitsbedingte Arbeitsunfähigkeit genüge getan werden kann, ohne dass es der Mitteilung einer konkreten Erkrankung bedarf. ${ }^{51}$ Von diesem Grundsatz wird man allerdings im Pandemiefall Ausnahmen zulassen müssen, ist doch der Gesundheitsschutz der übrigen Arbeitnehmer in die Abwägung mit den Persönlichkeitsrechten des infizierten Arbeitnehmers einzustellen. Demnach ist der Arbeitnehmer auf Grundlage seiner Rücksichtnahmepflichten ( $\$ 241$ II BGB, $\$ \$ 15,16$ ArbSchG) dazu verpflichtet, den Arbeitgeber über eine diagnostizierte Coronaerkrankung jedenfalls dann aufzuklären, wenn er für die Dauer der Inkubationszeit von 14 Tagen persönlichen Kontakt zu anderen Arbeitnehmern hatte. ${ }^{52}$ Auskuntt kann der Arbeitgeber weiterhin darüber verlangen, ob sich der Arbeitnehmer in den vergangenen 14 Tagen in Gebieten aufgehalten hat, in denen eine erhöhte Infektionsgefahr bestand, sowie darüber, ob der Arbeitnehmer in dieser Zeit Kontakt zu Personen hatte, bei denen ein begründeter Verdacht einer Infektion oder gar eine positive Diagnose besteht. ${ }^{53}$ Dem vergleichsweise geringen Eingriff in das Persönlichkeitsrecht des Arbeitnehmers steht hier ein schutzwürdiges Interesse der Belegschaft an Gesundheitsschutz gegenüber, hinter welchem die Interessen des betroffenen Arbeitnehmers ausnahmsweise zurückzutreten haben. Aus diesem Grund ist die hiermit verbundene Erhebung personenbezogener Daten nach Art. 6 I lit. c) DSGVO gerechtfertigt. ${ }^{54}$

Fraglich ist, inwieweit dem Arbeitgeber das Recht zusteht, die Gesundheit seiner Arbeitnehmer selbständig zu überprüfen, z.B. in Form von Fie-

51 ErfK/B. Reinhard, $\$ 5$ EFZG Rn. 5; U. Preis, Individualarbeitsrecht, Rn. 2156.

52 M. Weber, ARP 2020, 120, 122; A. Sagan/M. Brockfeld, NJW 2020, 1112.

53 M. Weber, ARP 2020, 120, 122; E. Dehmel/N. Hartmann, BB 2020, 885, 887; A. Sagan/M. Brockfeld, NJW 2020, 1112.

54 E. Dehmel/N. Hartmann, BB 2020, 885, 887; A. Sagan/M. Brockfeld, NJW 2020, 1112. 
bermessungen beim Betreten des Betriebsgeländes. Dabei muss berücksichtigt werden, dass - in Ermangelung flächendeckend einsatzbereiter Corona-Schnelltests - Untersuchungsmaßnahmen des Arbeitgebers wie Fiebertests nur einen begrenzten Aussagegehalt über eine tatsächliche Erkrankung liefern können. Vor dem Hintergrund dieser diagnostischen Unschärfe wird eine anlasslose Überprüfung sämtlicher Arbeitnehmer im Zweifel ausscheiden müssen. ${ }^{55}$ Etwas anderes kann aber gelten, wenn es im Betrieb bereits Verdachtsfälle oder gar diagnostizierte Infektionen gibt, da sich das Risiko einer betriebsinternen Ausbreitung des Virus hierdurch deutlich erhöht. ${ }^{56}$

\section{Aufklärungspflichten des Arbeitgebers}

Auf der anderen Seite trifft den Arbeitgeber eine Pflicht, die Betriebsangehörigen über Verdachts- und Infektionsfälle in der Belegschaft aufzuklären. ${ }^{57}$ Dabei ist allerdings dem Persönlichkeitsrecht des betroffenen Arbeitnehmers hinreichend Rechnung zu tragen, was eine Namensnennung unzulässig macht. ${ }^{58}$ Insoweit beschränkt sich die Aufklärungspflicht auf die Mitteilung darüber, dass ein Verdachts- oder Infektionsfall im Betrieb eingetreten ist; soweit hierdurch keine Rückschlüsse auf die Person des Betroffenen gezogen werden können, wird besonders bei großen Betrieben eine Konkretisierung auf einen abgrenzbaren Betriebsteil geboten sein.

\section{E. Die Krisensicherheit des Arbeitsvertragsrechts in der Pandemie}

Auf Grundlage dieser Risikoanalyse soll im Folgenden die Frage beantwortet werden, ob das Arbeitsvertragsrecht in der Coronakrise die notwendigen Instrumente bereit hält, um eine interessengerechte Verteilung der pandemiebedingten Risiken des Beschäftigungsmarktes zu ermöglichen. Dabei soll der Blick - auch wenn dies in Anbetracht der fortdauernden Infektionslage verfrüht erscheint - auch auf Entwicklungsperspektiven zur Vorbereitung künftiger Pandemiefälle gerichtet werden.

55 A. Sagan/M. Brockfeld, NJW 2020, 1112, 1113; ähnlich M. Fublrott, GWR 2020, 107, der eine generelle Duldungspflicht für Messungen am Betriebseingang von einem weiteren Anstieg der Fallzahlen abhängig machen will.

56 A. Sagan/M. Brockfeld, NJW 2020, 1112, 1113.

57 M. Weber, ARP 2020, 120, 123.

58 M. Weber, ARP 2020, 120, 123. 


\section{Das Arbeitsvertragsrecht als Korridor interessengerechter Krisenbewältigung}

Obwohl dem deutschen Arbeitsvertragsrecht der Begriff einer Pandemie fremd ist, zeigt die Analyse der vertraglichen Risikoverteilung, dass es für die mit der Coronakrise verbundenen Leistungsstörungen angemessene und interessengerechte Regelungen bereithält. Trotz der Neuartigkeit krisenbedingter Problemlagen wie dem Fehlen einer öffentlichen Kinderbetreuungsinfrastruktur und präventiven Betriebsschließungen bieten auslegungsfähige Tatbestände hinreichend Spielraum für die Berücksichtigung der gegenwärtigen Situation bei der vertraglichen Risikoverteilung. Bestehende Regelungslücken wie das Problem des Verdienstausfalls bei Erfüllung von Betreuungspflichten hat der Gesetzgeber dabei zügig geschlossen. Insgesamt muss sich das Arbeitsrecht ohnehin nicht an der Zielvorgabe messen lassen, eine abstrakte Best-Practice für sämtliche Arbeitsverhältnisse im Pandemiefall vorzuhalten, was in Anbetracht der Diversität von Beschäftigungsverhältnissen eine Herkulesaufgabe darstellte. Seine Aufgabe besteht vielmehr darin, den Arbeitsvertragsparteien einen Handlungskorridor vorzugeben, innerhalb dessen sie anhand ihrer individuellen Bedürfnisse und der Eigenarten der Tätigkeit angemessene Lösung finden können, ohne dass eine Partei ihre Interessen einseitig auf Kosten der anderen durchzusetzen vermag.

Dabei wird es besonders wichtig sein, bei der Auslegung des Arbeitsrechts die gegenseitige wirtschaftliche Abhängigkeit der Arbeitsvertragsparteien in ihrer gewerblichen Wertschöpfung hinreichend zu berücksichtigen. Das Risiko einer betrieblichen Existenzgefährdung schwebt in der Pandemie wie ein Damoklesschwert nicht nur über dem Kopf des Arbeitgebers, sondern auch der gesamten Belegschaft, werden die Arbeitnehmer in aller Regel von ihrem Arbeitsverhältnis als zentrale Quelle ihres Lebensunterhaltes abhängig sein. Beide Parteien verfolgen daher das gemeinsame Interesse, die Krise möglichst ohne größere wirtschaftliche Schieflage des Betriebes zu überstehen, um das Arbeitsverhältnis mit Blick auf die Zukunft normal weiterführen zu können. Vor diesem Hintergrund wird es in der Praxis geboten sein, individuelle Lösungen auf Basis von Kompromissen zu schaffen, anstatt auf dem Streitweg die Grenzen des Arbeitsvertragsrechts gerichtlich ermitteln zu lassen. ${ }^{59}$ Unterstützt werden die Vertragsparteien dabei durch den Gesetzgeber, welcher das wirtschaftliche Pande-

59 In diesem Sinne auch A. Sagan/M. Brockfeld, NJW 2020, 1112, 1117 unter Verweis auf die Bundeskanzlerin, die in ihren Ansprachen während der Coronakrise regelmäßig zu gemeinsamen solidarischen Handeln aufruft. 
mierisiko durch flankierende öffentlich-rechtliche Maßnahmen wie die Erleichterungen der Regelungen zum Kurzarbeitergeld abzumildern versucht.

\section{Die arbeitnehmerähnlichen Selbständigen als Sorgenkinder der Pandemie}

Lässt sich dem Arbeitsvertragsrecht hinsichtlich seiner Regelungsinhalte und -dichte ein ordentliches Zeugnis ausstellen, so wirft die gegenwärtige Krise doch zumindest Fragen hinsichtlich seines persönlichen Anwendungsbereiches auf. Denn es sind besonders die arbeitnehmerähnlichen Personen, die von den arbeitsrechtlichen Schutzmechanismen weitgehend ausgenommen sind, obwohl ihr wirtschaftliches Schicksal in der Pandemie regelmäßig eng mit dem ihres Dienstherrn verknüpft ist. Als Selbständige tragen sie die pandemiebedingten Wirtschafts- und Betriebsrisiken selbst, müssen ihre Tätigkeit auf eigene Kosten an besondere Verhaltensvorschriften anpassen und genießen gegenüber ihrem Dienstherrn keinen besonderen Kündigungsschutz. Den besonderen Schutzbedarf Solo-Selbständiger hat auch die Bundesregierung erkannt und bezuschusst daher Kleinstunternehmer, welche infolge der Coronakrise unter wirtschaftlichen Schwierigkeiten leiden. ${ }^{60}$

Die Notwendigkeit staatlicher Unterstützungsmaßnahmen wirft aber zumindest die Frage nach der wirtschaftlichen Verantwortlichkeit von Dienstherren jedenfalls in solchen Absatz- und Vertriebssystemen auf, die auf dem Verbund zahlreicher Einzelunternehmer unter einer gemeinsamen Marktpräsenz beruhen, wie dies z.B. bei Franchisesystemen oder Crowdworkplattformen der Fall sein kann. Während die System- oder Plattformbetreiber hier in wirtschaftlich stabilen Zeiten von Umsatzbeteiligungen und einem bedingt durch die stärkere Marktdurchdringung steigenden Markenwert von der gemeinsamen Wertschöpfungskette profitieren, sind sie gegenüber ihren Dienstleistern in der Krise über das für Dienstverträge gewöhnliche Maß hinaus kaum zu besonderer Unterstützung verpflichtet. Umgekehrt - und das zeigt mit der Vapiano-Insolvenz bereits die erste pandemiebedingte Zahlungsunfähigkeit eines Systemunternehmens - sind die selbständigen Dienstleister besonders in der Gastro-

60 So können kleine Unternehmen, Selbständige und Freiberufler für einen Zeitraum von drei Monaten Betriebskostenzuschüsse beantragen, welche vom Bund im Umfang von 50 Milliarden Euro bereitgestellt und von den Ländern ausgezahlt werden; daneben wurde auch der Zugang zur Grundsicherung für Selbständige erleichtert, um deren Lebensunterhalt und Wohnkosten abzusichern. 
nomiebranche jedenfalls mittelbar betroffen, wenn sich infolge der Krise das Wirtschaftsrisiko ihres Dienstherrn realisiert und dieser seinerseits zahlungsunfähig wird. ${ }^{61}$ Neben zu befürchtenden Umsatzeinbußen durch die Beeinträchtigung des systemeigenen Goodwills besteht hier die Gefahr des Verlusts der Berechtigung zur weiteren Marken- und Know-how-Nutzung. Im Ergebnis tragen solche Systemdienstleister somit die wirtschaftlichen Risiken sowohl ihres eigenen Betriebes als auch jene des Systembetreibers, ohne dass dem eine besondere vertragliche Verantwortlichkeit des Letzteren gegenübersteht. Dass der Staat dieses Schutzdefizit erkannt hat und ihm mit finanziellen Hilfsmaßnahmen entgegenzusteuern versucht, mag zwar aus wirtschaftspolitischer Sicht löblich sein. Die Erforderlichkeit solcher Maßnahmen impliziert jedoch eine Unverhältnismäßigkeit der Verteilung wirtschaftlicher Chancen und Risiken in Beschäftigungsverhältnissen, in denen der Dienstleistende in Ermangelung eines hinreichenden Weisungsniveaus zwar nicht persönlich, wohl aber wirtschaftlich von seinem Dienstherrn abhängig ist. Vor diesem Hintergrund können Zweifel daran aufkommen, ob es tatsächlich allein die persönliche Abhängigkeit des Beschäftigten ist, die den besonderen Schutzbedarf vollumfänglich begründet, welchen das Arbeitsvertragsrecht mit seinen Arbeitnehmerschutzvorschriften zu erfüllen bezweckt.

\section{Die Pandemie als (verpasste) Chance für den Beschäftigungsmarkt}

Zweifel an der Reichweite des geltenden Arbeitnehmerbegriffs können infolge der Coronakrise aber auch dadurch aufkommen, dass der Beschäftigungsmarkt unter dem Einfluss der Pandemie erstmals dazu gezwungen ist, in größerem Maße auf digitale Formen der Zusammenarbeit auszuweichen. Während sich diese Arbeitsformen - trotz ihrer mitunter überstürzten Einführung - in der Praxis als durchaus funktionstauglich erweisen, gehen sie doch mit einer schwindenden Kontrolle des Arbeitgebers über

61 Für die besonders gefährdeten Unternehmen des Gastronomiegewerbes erwägt die Bundesregierung eine befristete Mehrwertsteuersenkung für ein Jahr ab dem 01.07.2020, um die finanziellen Einbußen der pandemiebedingten Schließzeiten zu kompensieren, vgl. Entwurf eines Gesetzes zur Umsetzung steuerlicher Hilfsmaßnahmen zur Bewältigung der Coronakrise (Corona-Steuerhilfegesetz), https:/ /www.bundesfinanzministerium.de/Content/DE/Gesetzestexte/Gesetze_Gesetzesv orhaben/Abteilungen/Abteilung_IV/19_Legislaturperiode/Gesetze_Verordnunge n/2020-04-30-Corona-Steuerhilfegesetz/1-Formulierungshilfe.pdf?_blob=publicat ionFile\&v=4 (zuletzt abgerufen am 06.06.2020). 
den Arbeitsprozess einher. Arbeitet der Arbeitnehmer infolge der Pandemie von zu Hause und stellt ihm der Arbeitgeber dabei sogar teilweise frei, wann er bestimmte Leistungen zu erbringen hat, stellt sich die Frage nach dem für den Arbeitnehmerstatus erforderlichen Grad der Weisungsgebundenheit, welche sich durch die digitale Distanz zwischen den Arbeitsvertragsparteien aufzulösen droht. Solange aber die Weisungsgebundenheit konstitutives Merkmal für die Reichweite des arbeitsrechtlichen Schutzschirms bleibt, besteht jedenfalls die Gefahr, Beschäftigte aufgrund ihrer neu gewonnen Freiheiten bei der Erbringung ihrer Arbeitsleistung aus dem Arbeitsrecht zu verlieren.

Insoweit wird spannend zu beobachten sein, welchen Einfluss die Erkenntnisse aus der Krise auf die Fragen der Flexibilisierung von Arbeitsverhältnissen haben werden. So dürfte gerade die Debatte um einen Anspruch auf Homeoffice infolge der Pandemie zusätzlich an Fahrt gewinnen. ${ }^{62}$ Besonders Pendler, die in der Krise wochenlang ohne größere Einbußen von zu Hause gearbeitet und dabei die Vorteile wegfallender Fahrzeiten zu genießen begonnen haben, dürften auch nach der Pandemie ein gestiegenes Interesse an größerer Arbeitsort und -zeitflexibilität haben. ${ }^{63}$ Insofern begründet die gegenwärtige Krise für den Beschäftigungsmarkt, neben den hiermit nicht zu relativierenden, wirtschaftlichen Gefahren, auch die Chance der praktisch erzwungenen Erkenntnis digitaler Entwicklungspotentiale, die nicht nur während der Pandemie, sondern vor allem auch über sie hinaus Möglichkeiten zur Verbesserung und Vereinfachung betriebsinterner Vorgänge bieten.

Eine weitere Chance der Coronakrise hat der Beschäftigungsmarkt allerdings bereits jetzt schon verpasst. So haben nur wenige Themen den arbeitspolitischen Diskurs der letzten zehn Jahre so sehr dominiert wie die Notwendigkeit der Weiterbildung von Arbeitnehmern, ganz besonders vor dem Hintergrund zunehmender Digitalisierung und dem hiermit verbundenen Erfordernis des Erwerbs informatorischer Kompetenzen. ${ }^{64}$ Die

62 M. Fublrott/K. Fischer plädieren insoweit ebenfalls für die Normierung der Grenzen einer Homeoffice-Pflicht, NZA 2020, 345, 350.

63 Laut einer Kurzbefragung des Bayrischen Forschungsinstituts für Digitale Transformation wünschen sich $68 \%$ der Arbeitnehmerinnen und Arbeitnehmer, die Homeoffice bei ihrer Tätigkeit grundsätzlich für möglich halten, auch nach der Coronakrise mehr Homeoffice als zuvor, R. Stürz et. al., Digitalisierung durch Corona?, https://www.bidt.digital/studie-homeoffice (zuletzt abgerufen am 06.06.2020).

64 Beispielhaft R. Giesen/J. Kersten, Arbeit 4.0, 2018, S. 40; zuletzt A. Bromme, in: Klawitter et. al. (Hrsg.), Assistententagung im Arbeitsrecht 9 (2019), S. $181 \mathrm{ff.}$ 
wochenlange häusliche Isolation und das beschränkte Leistungsinteresse von Arbeitgebern hätten - entsprechende digitale Weiterbildungsinfrastrukturen vorausgesetzt - den Nährboden für eine Weiterbildungsoffensive bereiten können. Auf diese Weise hätten Arbeitgeber ihre wirtschaftlichen Verluste der Krise durch einen unternehmensinternen Know-howGewinn zwar nicht wettmachen, jedenfalls aber teilweise kompensieren können. Mit Blick auf die Möglichkeit künftiger Pandemiefälle, die in Anbetracht der fortschreitenden Globalisierung zunehmend als wirtschaftlicher Risikofaktor zu berücksichtigen sein werden, sollten daher nach der Krise staatliche Maßnahmen erwogen werden, die Anreize zu einer betrieblichen Pandemieplanung insbesondere unter Berücksichtigung eines internen Weiterbildungsbedarfes setzen.

\section{F. Fazit}

Zusammenfassend lässt sich sagen, dass das Arbeitsvertragsrecht in der Coronakrise gut aufgestellt ist, um pandemiebedingte Vertragsrisiken angemessen und interessengerecht zwischen den Parteien zu verteilen. Die auslegungsfähigen Tatbestände arbeitsrechtlicher Vorschriften bieten hinreichend Raum für die Berücksichtigung der individuellen Bedürfnisse der Arbeitsvertragsparteien im konkreten Arbeitsverhältnis. Dabei unterstützt der Gesetzgeber den Beschäftigungsmarkt einerseits durch Schließung etwaiger Regelungslücken, andererseits durch finanzielle Hilfsmaßnahmen, die die drohenden Wirtschaftsrisiken abzumildern versuchen. Weniger Glück haben an dieser Stelle die arbeitnehmerähnlichen Personen, die zwar in aller Regel ähnlich betroffen von den Risiken der Pandemie sind wie gewöhnliche Arbeitnehmer, im Verhältnis zur ihrem Dienstherrn dabei aber kaum besonderen Schutz genießen. Ihnen greift zwar der Staat finanziell unter die Arme, um die pandemiebedingten Ertragsausfälle teilweise zu kompensieren, das Arbeitsvertragsrecht wird ihnen in der Krise hingegen regelmäßig nicht helfen. In Anbetracht der erzwungenen Entgrenzung der Arbeit aus dem Betrieb als potentiellem Infektionsherd wird zudem spannend zu beobachten sein, wie sich infolge der Coronakrise der arbeitsrechtliche Diskurs insbesondere in Bereichen wie der Flexibilisierung von Arbeit durch den Einsatz digitaler Kommunikationswege oder auf dem Feld der Arbeitnehmerweiterbildung entwickeln wird. 